\title{
Propuesta de gestión compleja para organizaciones como estructuras disipativas
}

\author{
Dr. Manuel Enrique Villalba Puerta \\ Universidad de La Salle (Colombia)
}

mvpvillalba@gmail.com

Recepción:26/05/2020

Aceptación:20/06/2021

Propósito: El propósito de este artículo responde a la continuidad de un proyecto de investigación que inició en el año 2011, y que se interesa en comprender la gestión y la organización desde las ciencias de la complejidad. Los objetivos se trazaron en la siguiente línea: 1) pensar la gestión desde las ciencias de la complejidad; y 2) pensar la organización como una estructura disipativa. Ahora bien, la continuidad con este artículo se da en 3) proponer un enfoque de gestión compleja para organizaciones como estructuras disipativas.

Diseño/Metodología/Enfoque: El enfoque del tema es de tipo exploratorio de las teorías de las ciencias de la complejidad y su implementación en el contexto de las organizaciones. Por lo tanto, el alcance se fundamenta en traslapar la teoría de las estructuras disipativas y fractales en la realidad organizacional.

Hallazgos: Se presenta una propuesta de gestión compleja para organizaciones disipativas.

Implicaciones prácticas y sociales: La gestión compleja es un sistema auto-eco-organizador, autónomo dependiente del medio que lo rodea. Por lo tanto, es una gestión que busca que prevalezca la Vida y su coevolución con los demás sistemas. En ese sentido, el cambio se encamina en comprender y entender que, la economía (consumismo exacerbado) no puede estar por encima de la Vida, es decir, la economía debe pivotear en torno de la Vida, no viceversa; dicho de otra manera, es una gestión que coloca todas las condiciones para que la Vida sea posible.

Originalidad: El valor del artículo consiste en presentar una nueva mirada de gestión y organización desde las ciencias de la complejidad. Donde la gestión y la organización se entiende como sistemas dinámicos, complejos, y alejados del equilibrio.

Financiamiento: No existe ningún tipo de financiación por parte de alguna institución pública o privada.

\section{Resumen}

Este texto presenta una propuesta de gestión compleja para organizaciones como estructuras disipativas. La tesis propuesta afirma que la gestión compleja es un sistema auto-eco-organizador, es decir, autónomo y dependiente del entorno que lo rodea. Por lo tanto, la gestión se convierte en una canalizadora de procesos que 
a través de la visión fractal ayuda a colocar todas las condiciones para posibilitar la Vida de la organización y su entorno.

\section{Palabras clave}

Gestión compleja, estructuras disipativas, fractales, entropía, Business Intelligence, estrategia.

\begin{abstract}
This text presents a proposal of complex management for organizations as dissipative structures. The thesis proposed here states that complex management is a self-eco-organized system that is autonomous and dependent of the environment that surrounds it. Therefore, management turns into a process of channeling that through fractal vision helps to put all the conditions to make life in the organization and its environment possible.
\end{abstract}

\section{Keywords}

Complex management, dissipative structure, fractal, entropy, Business Intelligence, strategy

Clasificación JEL (Journal of Economic Literature): Q57M29

\section{Introducción}

Proponer una gestión compleja para organizaciones disipativas es un trabajo que se viene adelantando desde el año 2011 con investigaciones orientadas a entender la gestión y la organización desde las ciencias de la complejidad. Los conceptos de gestión y organización que se remontan hasta la década de los cincuenta, enfocados en sistemas de planificación, y que se han usado hasta hoy, responden a una lógica cartesiana-lineal donde priman la predictibilidad y el determinismo. Teóricos de la gestión como Fayol y otros desarrollaron mecanismos de control basados en este paradigma para pensar la organización como una máquina, en donde la gestión administrativa incorpora planes que se desarrollan en la lógica de la planificación, presupuestos y sistemas de gestión a partir de objetivos, para luego disponer de estructuras centralizadas y burocráticas, propiciar la división del trabajo, desarrollar procesos de auditoría e implementar mecanismos de estandarización para mantener el equilibrio.

En este sentido, son pocos los que entienden que la organización ha evolucionado de un estado rígido a uno flexible. Un ejemplo de ello son los cambios que ha generado la COVID-19 en el (los) comportamiento(s) de los individuos y su relación con el entorno, que ha obligado a las organizaciones a desarrollar procesos adaptativos. De ahí que las habilidades de liderazgo y de gestión empiecen a evolucionar para hacer frente a un entorno cada vez más complejo. Pensar la gestión compleja es dejar de trabajar sobre planes, guías y fórmulas y pasar a trabajar en escenarios dinámicos, borrosos, de emergencia, de no-equilibrio y de incertidumbre, con el propósito fundamental de absorber la complejidad (acciones adaptativas).

El desconocimiento de la complejidad del sistema tiene a la organización jerárquica actual en crisis, pues la complejidad de acción (interacción-comportamientos) exigida por la organización social ha superado la del propio sistema. Por consiguiente, querer ser eficaces en un mundo rápido y complejo obliga a la organización a ser rediseñada para que la información se transmita de forma horizontal, es decir, que las redes de interacción reemplacen el control jerárquico (Bar-Yam, 1997) y el resultado de estas interacciones no-lineales entre los componentes hagan que el sistema sea emergente; entonces se trata de un sistema complejo (Maldonado Castañeda, 2003). Un ejemplo de esto es la actual interacción de los individuos en todo el mundo que hace que las actividades diarias sean más complejas de lo que nunca habían sido. Este aumento de la complejidad global obliga a que las organizaciones humanas sean igualmente complejas y los avances tecnológicos ayuden a que las organizaciones pasen del control centralizado al control repartido (Bar-Yam, 2002).

Por lo anterior, a fin de presentar una propuesta de gestión compleja para organizaciones como estructuras disipativas, procedo a través de dos (2) pasos así: en un primer paso, presentaré la visión de organización como estructura disipativa donde los niveles de energía se disipan hacia puntos de bifurcación que logran la evolución del sistema. En un segundo paso, presentaré la visión de gestión compleja, que desde la fractalidad entiende que una parte contiene el todo y con estrategias, como autómata celular, busca absorber la complejidad de la organización para una mayor adaptación y sensibilidad al medio.

\section{Organización como estructura disipativa}

La organización como estructura disipativa consiste en entender que el desorden es generador de orden y que su estructura está alejada del equilibrio porque gravita permanentemente con el intercambio de información, materia, y energía. Es un sistema en donde confluyen múltiples lógicas y diversidad de fuerzas que conllevan a la organización a escenarios armónicos de un orden 
creativo. En otras palabras, mientras la organización busca el equilibrio, su estructura y los elementos que la conforman se mantienen en conflicto, ambigüedad, aprendizaje y diálogo, acciones e interacciones que están fuera del equilibrio. Como dice Prigogine "[...] son islas de orden en un mar de desorden" que aumentan su orden a expensas del creciente desorden del entorno, es decir, organizaciones que viven procesos de ambigüedades y dualidades. En tales casos, "[...] el equilibrio no es posible, por darse procesos disipativos que continuamente producen entropía" (Prigogine y Stengers, 1983).

En ese sentido, la organización disipativa no es solo un sistema abierto que se alimenta de flujos que lo mantienen lejos del equilibrio, sino también es un sistema que se informa de manera selectiva de pequeñas diferencias que en el equilibrio son insignificantes (Prigogine y Stengers, 2004), pero que en el caos son generadoras de puntos de bifurcación. Estos puntos le permiten a la organización evolucionar del status quo a una estructura más compleja, así como "[...] los ecosistemas evolucionan por desorganizaciones y reorganizaciones. Las eras se suceden a través de una dialéctica de innovación, accidentes, catástrofes" (Morin, 1993).

Desde luego, esta es una organización que evoluciona para reconocer los fenómenos socio-eco-ambientales, porque entiende que sus interacciones están influenciadas por las necesidades y expectativas del entorno y del medio ambiente. En otras palabras, es una organización estructurada como "sistema viviente", que destaca la capacidad del propio sistema (autoorganización) para recrear las condiciones necesarias y lograr continuidad y adaptación a los cambios ambientales de una sociedad de mercado (Morris B, 2003). Para esto se necesita que las organizaciones piensen en la vida y en alternativas que promuevan la vida, tanto de los individuos como de los sistemas que los rodean (una posibilidad con relación a lo existente), y que comprendan la dependencia entre sistemas, que ayuda a la organización a coevolucionar a mayores y mejores condiciones de vida en contextos cada vez más complejos. En síntesis, una organización así es dinámica porque presenta cambios y evoluciona a estados más sensibles y adaptativos con respecto al medio ambiente, y es inestable porque presenta fluctuaciones (interacciones) que generan puntos de bifurcación en donde el sistema se adapta para luego evolucionar.

A continuación, se presentan las características de la organización disipativa, así: 1) pequeñas perturbaciones (fluctuaciones) precipitan al sistema hacia otros estados; 2) pequeñas causas producen efectos desproporcionados; y 3) la no proporcionalidad en los cambios de los procesos no lineales genera pérdida de unicidad de soluciones, es decir, hay más de una solución posible por los mismos grados de complejidad que tiene la organización. Como dice Cornejo Álvarez (2004), la organización maneja tipos de complejidad, como:

Complejidad de origen. Surge de la interacción con los demás sistemas en donde la tecnología juega un papel de administración de la complejidad. Un ejemplo: el manejo de big data a través del Business Intellingence $(\mathrm{BI})^{1}$.

Complejidad residual. Surge del resultado de los conflictos y del descontrol de los procesos. Un ejemplo: Ios procesos de resolución de conflicto de manera positiva en donde la comunicación se convierte en un camino de posibilidades.

Complejidad provocada. Surge de los procesos de toma de decisiones inconsistentes. Un ejemplo: la toma desinformada de decisiones, sin reconocer el medio y el entorno que rodea la organización y que hace que el proceso sea inconsistente.

Por lo anterior se puede decir que son los tipos de complejidades los que hacen de la organización disipativa un sistema que vive en entropía (segunda ley de la termodinámica), donde la energía aumenta en la medida que progresa el comportamiento de los fenómenos, que se disipa por sus tensiones. Es ahí donde la organización pasa del orden al desorden, es decir, a un orden más estructurado.

Se trata de una estructura que emerge y tiene sus propias partículas, propiedades y características, y que abre caminos a escenarios de incertidumbre donde existen posibilidades de desarrollo para la organización. Dicho de otra manera, es una estructura persistente y definitoria de su comportamiento que ayuda al sistema a encontrar su propia forma. Un ejemplo son los equipos de trabajo que tienen la capacidad de manejar la redundancia de variables y/o variedad mínima requerida, así como también las especificaciones mínimas críticas a través de la autogestión.

De otra parte, es importante traer a colación lo mencionado renglones atrás frente al nivel de dependencia de la organización con respecto a los demás sistemas. Justamente acá cabe mencionar que la organización disipativa no puede estar sujeta a relaciones netamente

1 Es un sistema que combina datos operativos con herramientas analíticas para presentar información; esto con el objetivo de ayudar a una toma de decisiones cercana a la realidad compleja. 
dependientes del crecimiento económico². En ese sentido, los puntos de bifurcación no pueden estar dados ni interpretados en el afán de la producción exacerbada. Dicho positivamente, es pensar la organización con una estructura sensible que se adapta a las necesidades del entorno específicamente, que responde a los efectos negativos que ha dejado la sociedad del progreso $^{3}$. En consecuencia, es una organización que concibe la vida no solo como un sistema de intercambio de información, materia y energía, sino más bien como el intercambio de transiciones entre cada una de ellas y con otras cosas (Kauffman, 2000), siendo los resultados de las transiciones las que muestran los comportamientos que confluyen entre ellas y que son la vida misma del sistema. Por lo tanto, el papel de la organización disipativa apoyada de la gestión compleja consiste en: 1) promover la existencia de unidades autorreguladas, pero orgánicamente integradas al sistema integrador (Vida); 2) transformar sus estructuras y dinámicas jerarquizadas en procesos (actividades); y 3 ) en la constante fluidez y transformación de niveles a multiniveles (Eschenhagen y Maldonado, 2014).

En síntesis, es una organización viviente que tiene la capacidad de aprender constantemente de los problemas y las oportunidades generadas por la dinámica del entorno, capaz de adaptarse y renovarse con nuevas habilidades y actitudes que ayudan a la transformación y evolución del sistema.

\section{Gestión Compleja}

Desde la perspectiva de la organización como estructura disipativa es posible pensar la gestión compleja. Esto se debe a que la realidad de muchas organizaciones, a pesar de varios períodos de éxito, ha entrado en crisis profunda por los enfoques tradicionales de gestión empresarial (Gil Domínguez, 2006) que no reconocen el dinamismo, la inestabilidad, la emergencia y la incertidumbre, características de los entornos complejos, y que tampoco han entendido que las organizaciones son conjuntos sociales, reconocibles e identificables, que se relacionan con un contexto (proveedores, clientes, ambiente y naturaleza) y para lo cual requieren desarrollar estructuras flexibles que les permitan adaptarse a las posibilidades de su entorno (Etkin, 2006).

2 Es una relación netamente reduccionista de la realidad Lo que se pretende con la organización disipativa es que las reglas de interdependencia prevalezcan sobre la competencia (Max-Neef, 1993).

3 Lo que se busca es cambiar la mirada y centrarse en el concepto de Bioeconomía propuesto por Georgescu Roegen. Aunque este texto no es el espacio de discusión para este tema, es necesario dejar claro que el papel de los seres humanos es establecer los valores y el papel de la naturaleza es establecer las reglas.
En otras palabras, son enfoques que tienen una mirada economicista y olvidan la incidencia de los fenómenos no económicos (medio ambiente, naturaleza) para el desarrollo de las organizaciones. Asimismo, ignoran los accidentes (cambios naturales), la creatividad y creen aprehender la naturaleza profunda de la realidad, en una concepción ciega a la naturaleza compleja de esta realidad (Morin, 1993).

El propósito de este artículo no es profundizar en los enfoques de gestión empresarial tradicional, ${ }^{4}$ sino proponer un nuevo enfoque de gestión que desde las ciencias de la complejidad permita acercar a la organización a caminar en entornos complejos. Se trata, por lo tanto, de un enfoque que ve la organización desde lo social, cultural, ambiental y natural, en donde no hay una razón excluyente, ni tampoco un pensamiento único o verdadero. Desde esta cosmovisión, la organización requiere más interacción, diálogo, discusión y redes de comunicación, antes que procedimientos rígidos o jerarquías formales (Etkin, 2006).

Esta es una visión de organización que hace que el sistema viva en desorden y a su vez se convierta en un generador de desequilibrio (no-equilibrio), es decir, pone en evidencia la necesaria coexistencia de orden y desorden. Esta relación, en términos de Hock (2001), conduce a la llamada "organización caórdica" que destaca la existencia de procesos de renovación o, según Prigogine, "bifurcaciones", es decir, procesos de evolución que se movilizan desde la tensión creativa.

Pensar el desorden como generador de orden es uno de los principios de las leyes del caos que introduce aspectos nuevos y esenciales, como la generación de un nuevo orden más sensible y coherente que se correlaciona con todas sus partes. En ese sentido, Prigogine descubre que de cierto alejamiento del equilibrio el orden emerge del caos, y las formas organizadas que surgen del caos sobreviven únicamente si se mantienen abiertas al flujo constante de materia y energía (Bondarenko Pisemskaya, 2007).

Desde esta perspectiva, la gestión compleja se convierte en un sistema no lineal, abierto, creativo y caótico, que puede organizarse y mantenerse por sí mismo. Un buen ejemplo es la internet, la cual se mantiene por un flujo abierto de usuarios y no es controlada ni manejada

$4 \quad$ Aquellos enfoques de gestión mecanicista-lineal que fragmentan la realidad y no reconocen los escenarios borrosos, azarosos, inestables, ambiguos y duales de la organización y el entorno. Estos son: gestión de desarrollo organizacional, gestión de calidad total, gestión de cambio, gestión estratégica, gestión de mercados, gestión de reingeniería de procesos, entre otros. 
por nadie. La gestión se deriva a partir del acoplamiento de la retroalimentación que surge de la actividad individual aleatoria y se mantiene gracias a la combinación de los elementos de competición y cooperación, procesos que se complementan y hasta se convierten el uno en el otro (Bondarenko Pisemskaya, 2007). Una vez traspasado el umbral de aleatoriedad, los sistemas se destruyen y emergen otros nuevos (Briggs, 2005), donde la gestión es creativa y se adapta al contexto.

En consecuencia de lo anterior, se puede decir que se trata de una visión compleja que destaca la capacidad del propio sistema (gestión) para recrear las condiciones necesarias y lograr continuidad y adaptación con respecto a los cambios ambientales de una sociedad de mercado, interpretada como sistema abierto, dinámico, complejo y adaptativo (Morris, 2003). Además, en la medida en que aumenta el comportamiento de los fenómenos, la energía disipada no se recupera, pero en la creciente entropía el sistema logra evolucionar.

A continuación, se presentan los factores básicos que se pueden asociar a la gestión compleja en la Tabla1.

Tabla 1

\section{Factores básicos de la gestión compleja}

\begin{tabular}{ll}
\hline $\begin{array}{l}\text { Referente } \\
\text { macrotextual }\end{array}$ & $\begin{array}{l}\text { Factores } \\
\text { básicos }\end{array}$ \\
\hline & $\begin{array}{l}\text { Comunicación } \\
\text { Estrategias }\end{array}$ \\
Gestión & Aprendizaje \\
& Cambio \\
& Adaptación \\
& Creatividad \\
\hline Elementos & Auto-eco-organizador \\
transversales & Interacción \\
& (comportamientos) \\
\hline
\end{tabular}

Nota: Esta tabla muestra los factores básicos de la gestión compleja. Elaboración propia.

Entender estos factores desde una realidad compleja es pensar en: 1) la consideración de la comunicación como un sistema autopoiético, 2) las estrategias como autómata celular, 3) el aprendizaje organizacional como un sistema vivo, 4) el cambio como eje de transformación, 5) la adaptación como un escenario de evolución, y 6) la creatividad como la oportunidad de crecimiento (coevolución). Todo esto es posible en la medida en que exista la interacción permanente (comportamiento) de los sistemas y, como dice Morin (1993), sistemas que son auto-eco-organizador, es decir autónomo/dependientes con respecto a sus ecosistemas.

Comunicación como un sistema autopoiético. Se parte de la base de que no se trata de un proceso lineal y que no se puede reducir a la relación causa-efecto. Esto se debe a que el ser humano siempre construye sentido -interpreta- desde su propio mundo, en un proceso multidimensional en el cual se dan varios procesos simultáneamente y son esenciales para la operación de una organización. Dicho de otro modo, una organización es un sistema comunicativamente cerrado porque produce comunicación mediante comunicación, no puede generarse independientemente de su entorno y se constituye al trazar sus operaciones (Luhmann, 2007). Además cuenta con procesos y procedimientos que son autorreferenciales porque operan por autocontacto, autocreación (auto $=$ sí mismo + poein $=$ producir o crear) (Porskein, 2004), en su relación con el entorno. También se puede comprender como una unidad continua de actualización y virtualización de posibilidades (Luhmann, 1998) que comunican no sólo a través del lenguaje, sino también por medio de la comunicación corporal como medio de expresión (símbolos simbióti$\cos )^{5}$.

Estrategias como autómata celular. Consiste en reconocer la(s) estrategia(s) como un autómata celular, es decir, en una organización disipativa la(s) estrategia(s) son susceptibles de asumir distintos estados, esto quiere decir que la(s) estrategia(s) mantiene(n) relaciones con sus aledaños y el estado que adopta(n) depende del tipo de relación que establezca(n), así como también con los estados de su vecindad. Este proceso se conoce con el nombre de "regla" o "función de transición" (Arroyo Méndez, Millán y Hassan Collado, 2007).

\section{Aprendizaje organizacional como un sistema vivo.}

Se da porque el sistema interactúa permanentemente con información, materia y energía, es decir, es un sistema que comprende la biología de su aprendizaje, porque reconoce que la convivencia, las experiencias e influencias del entorno impactan la información de su estructura celular. En ese sentido, es un sistema que vive interactuando y en constante aprendizaje, en donde el conocimiento va formando cambios en la estructura del sistema, de modo que el aumento del conocimiento representa una ampliación del sistema y su reorganización hace posible nuevas formas de acción y nuevos resultados (Bateson, 1993).

$5 \quad$ Es la representación perceptible de una idea por rasgosasociados por una convención socialmente aceptada. 
Por todo ello se puede decir que el aprendizaje es un fenómeno que se da permanentemente y en él participan la historia, el lenguaje, la cultura y el clima, pero no desde la lógica de acumulación de saberes, sino como una red o trama de interacciones complejas que van creando estados cualitativamente nuevos en la estructura del sistema (Assmann, 2002). Es acá donde nace la oportunidad para que la gestión compleja se convierta en una facilitadora y/o catalizadora de procesos porque cuenta con la capacidad de procesar la información para buscar mayor autonomía, en otras palabras, coloca todas las condiciones para que el enfoque auto-eco-organizador ocurra y aproveche sus ventajas (Villalba Puerta, 2012). Una gestión que desde los procesos autorreferenciales (identidad sistémica) logra tener una relativa autonomía en sí misma, porque la flexibilidad estructural juega un papel fundamental, otorgando adaptabilidad al sistema en conexiones permanentes hacia dentro y hacia fuera, pero siempre condicionados por el entorno (Assmann, 2002).

Cambio como eje de transformación. El cambio se comprende en el sentido de reconocer que la realidad es cambiante, que lo nuevo puede surgir, que de todas maneras va a surgir y que, por lo tanto, el cambio organizacional debe buscarse en la trama interna del propio sistema, el cual no responde siempre de la misma manera por influjo de los factores externos que lo condicionan. Este cambio es posible por los procesos de autoorganización que ayudan a desarrollar la capacidad de respuesta del sistema hacia estímulos de una realidad de cambio. Así ocurre, por ejemplo, cuando hay un proyecto común, para el cual se da la posibilidad de trabajar bajo una integración de red y como fruto de esto, nacen equipos de trabajo entre áreas que ayudan al desarrollo del proyecto, posibilitando el cambio. De ahí se desprende la capacidad de responder desde la diversidad de soluciones para asegurar la aceptación de oposiciones y el no equilibrio en los resultados. ${ }^{6}$

En síntesis, el cambio, según Aristóteles, es un sistema "inteligible" que encuentra en la dinámica interna aspectos asociados a los procesos morfogenéticos que producen las formas invariantes del sistema, es decir, la causa eficiente es la forma operando del sistema, en donde la forma actúa como una fuente de cambio (Maldonado Catañeda, 2005).

Adaptación. Se entiende como una característica propia de las estructuras disipativas en el contexto de organizaciones que lejos del equilibrio logran espon-

6 Tomado en clase: Maestría en estudios y gestión del desarrollo, tema: "¿Qué significa una organización compleja?", profesor Raúl E. Nieto, consultor de cambio. Universidad de La Salle, Bogotá -Colombia. táneamente nuevas estructuras. Una organización en donde confluyen un sinnúmero de variables (ambientales, sociales, económicas, políticas) y fluctuaciones (interacción), las cuales son cada vez más grandes y llevan a la organización a puntos de bifurcación en donde el sistema presenta correlaciones entre los acontecimientos y se comporta como una totalidad (nace una nueva estructura). En palabras de Manrique Solana (1987), el sistema cumple la lógica de adaptación en yuxtaposición a los modos de creatividad del sistema para lograr su nueva estructura.

Creatividad. La creatividad se manifiesta en un flujo de energía que integra emociones, pensamientos (ideas) y acciones. Esta conjugación genera unos grados de vibración que ayudan a pasar a la organización del status quo a un estado más complejo donde el sistema evoluciona (Cabreras Cuevas, 2018). Por lo tanto, la creatividad evoluciona hacia formas progresivamente más complejas por la necesidad misma del sistema y la permanente interacción entre los componentes psicológicos, ambientales y los efectos de la creatividad sobre sí misma. Dicho de otro modo, la creatividad innovadora se da en campos de energía que transforman el valor humano desde lo social y lo ético, todo acompañado de conciencia (Cabrera Cuevas, 2009).

Luego de conocer los factores que se asocian a la gestión compleja paso a los principios que la gobiernan. Estos son, según Morín: 1) principio dialógico, 2) principio de recursividad organizacional y 3 ) principio hologramático.

Principio dialógico. Se da cuando el sistema desarrolla las aptitudes para tolerar, captar, integrar y transformar el desorden (Morin, 2006) en escenarios donde los procesos informales generan dualidad en los procesos jerárquicos y estos procesos se convierten en generadores de valor para el sistema. Dicho negativamente, son organizaciones jerárquicas que desconocen la naturaleza, las necesidades y las potencialidades del lado social (trabajadores) y buscan intereses particulares. Dicho positivamente, son organizaciones jerárquicas que logran alinear los objetivos de las personas y del (los) sistema(s) ${ }^{7}$ en un conjunto coherente de intereses complementarios que hacen que la organización evolucione (Hernandez Santana, 1997).

Principio de recursividad. Se entiende como un proceso en el que los efectos o productos son a su vez causantes y productores del proceso mismo, y en el que los estados finales son necesarios para la generación de los iniciales. De este modo, el proceso recursivo se pro-

7 Describe los sistemas sociales humanos, sistemas naturales y sistemas artificiales. 
duce/reproduce a sí mismo, evidentemente a condición de ser alimentando por una fuente, una reserva o un flujo exterior (Morin, 2006).

En ese sentido, la organización es el resultado de su propia actividad y esa actividad constituye en sí misma la organización. Un ejemplo: la organización manufacturera (zapatos) es igual a zapatos y la organización ferroviaria es igual a trenes. De esta manera, la gestión compleja reconoce las fuentes desde la recursividad, las reservas y los efectos del exterior como resultado de la actividad de una organización. Por lo tanto, esta mirada integradora posibilita que exista una preocupación por los efectos negativos (deterioro ambiental) a causa de la actividad económica y enfoca sus esfuerzos en diseñar estrategias para mitigar el impacto, al tiempo que permite que los sistemas puedan coevolucionar ${ }^{8}$.

Principio hologramático. Expresa que las partes constituyen un todo, es decir, que la parte podría ser más o menos apta para regenerar el todo (Morin, 2006). En este sentido, las partes pueden estar dotadas de relativa autonomía, pueden tener comunicaciones entre sí, lo que hace que contengan el todo. En la gestión compleja el sentido hologramático se da en la medida en que los procesos de buenas prácticas se pueden replicar en contextos internos y externos a la organización en donde se canaliza la información y se ejecutan las actividades.

En ese orden de ideas, la gestión compleja tiene la capacidad de procesar información para buscar mayor autonomía y a la vez sensibilidad con el medio ambiente en la medida en que centra su mirada 1) en la gestión de la incertidumbre, 2) en la gestión de la información y de significados, 3) en el apoyo a fuerzas contrapuestas y la utilización de conflictos positivos, y 4) en la gestión centrada en valores nucleares y borrosos. Esta visión posibilita dejar las estrategias a largo plazo porque entiende que están sometidas al azar, a las fluctuaciones y a las bifurcaciones del entorno y que en el umbral de complejidad la organización sigue rumbos imprevisibles, donde tiene cabida la incertidumbre como factor de choque, por lo que es capaz de reconocer las actividades creativas que en la inestabilidad expresan caminos de posibilidades. Es ahí donde la gestión toma fuerza en el diseño de estrategias adaptativas que se adecúan a los cambios del entorno y, como dice Zimmerman (1993), es más importante gestionar intentando absorber la incertidumbre que gestionar la propia incertidumbre.

8 Se entiende como un sistema que es recíproco en su proceso evolutivo. Es decir, que nada puede existir aisladamente, nada puede evolucionar sin la coexistencia con otros sistemas.
Por lo anterior, se puede decir que la información juega un papel fundamental, que a través de un sistema de "Business Intelligence" (BI) recopila y almacena datos en tiempo real, destaca alertas proactivas (creativas) y detecta anomalías e información geográfica como insumo de análisis para la toma de decisiones (Negash, 2004). En otras palabras, la información construye escenarios de posibilidades (rutas) que con fuerzas contrapuestas (diferencia) y la utilización de conflictos positivos se suma a la robustez de la decisión y se convierte en una herramienta clave para que la organización se ajuste al medio (Nonaka y Takeuchi, 1999). En hilo, se puede decir que es una decisión que se fundamenta en valores borrosos y que reconoce más valores de veracidad y falsedad que verdadero o falso. Ejemplo: un día soleado puede ser $100 \%$ verdad si no hay nubes; $80 \%$ verdad si hay pocas nubes; $50 \%$ verdad si existe neblina; y $0 \%$ verdad si el cielo está totalmente cubierto (Lozano y Fuentes, 2008).

En síntesis, la gestión compleja reconoce que no existe una solución individual sino que existen múltiples soluciones; en otras palabras, existen varias formas de ver las cosas, y cada cosa tiene su propia solución (Villalba Puerta, 2012). Por lo tanto, pensar la estrategia como curso de acción ayuda a la gestión a enfrentarse a escenarios que comportan incertidumbre y eventualmente peligros, en donde la estrategia puede modificar sobre la marcha el guión de acción en función de la información, reacciones, albures, acontecimientos, apariciones o suspensiones inesperadas de obstáculos, y se enriquece en experiencias y en aptitudes para responder a la diversidad (Villalba Puerta, 2012).

A continuación, se presenta una propuesta de gestión compleja que desde la fractalidad que busca responder a las necesidades de un entorno cada vez más complejo. Por tanto, es importante traer a colación algunas características de los fractales para entender su dimensión.

Autosimilaridad. Al mirar una parte de la organización a mayor escala parece igual y no se distingue el cambio de escala. En algunos casos se da de forma exacta y en otros no.

Dimensión fractal. Quiere decir que la dimensión fractal es mayor a su dimensión topológica?.

Diferenciales en los puntos. Esto quiere decir que los puntos son diferenciales el uno del otro, es decir, no

9 Se entiende por dimensión topológica el estado natural de una cosa. En este caso, para la propuesta de gestión compleja se trabajará bajo la dimensión topológica de un cuadrado $=2$. 
son idénticos no se pueden repetir (Sáenz de cabezón, 2015).

Por lo tanto, en el ejercicio de traslapar la visión fractal en la gestión compleja se puede decir que las "acciones" que se desarrollan al interior y exterior de la organización describen igualmente a la organización en términos de su naturaleza. Un ejemplo: una organización (ONG) a través de acciones filantrópicas describe la naturaleza como organización. En ese sentido, y en la búsqueda de entender la dimensión fractal, es importante comprender que el cambio de escala lo da el número de variables (dimensión fractal) que interactúan con la organización, de manera que, sin importar el número de variables, la organización sigue igual. Por consiguiente, la gestión compleja entiende la dimensión fractal (variables) como la oportunidad de implementar estrategias como autómata celular, que en los distintos estados responde a los puntos diferenciales y que en su gran mayoría no son idénticos. En otras palabras, son estrategias que responden a los posibles resultados de interacción (comportamientos) de la organización con su entorno.

Dado lo anterior, y a modo de entender con un ejemplo la gestión compleja, en esta oportunidad se utiliza la lógica de "conteo de cajas" para comprender un poco más la dimensión fractal10.

\section{Fórmula:}

\section{Dimensión topológica $=$ Dimensión de origen $\mathbf{x}$ dimensión factor}

$$
\begin{aligned}
& 2=5 * 2=10 ; 2=10 \\
& 2=5 * 3=15 ; 2=15 \\
& 2=5 * 4=20 ; 2=20 \\
& 2=5 * 5=25 ; 2=25 \\
& 2=5 * 6=30 ; 2=30 \\
& 2=5 * 7=35 ; 2=35
\end{aligned}
$$

\section{Figura 1}

\section{Dimensión fractal}

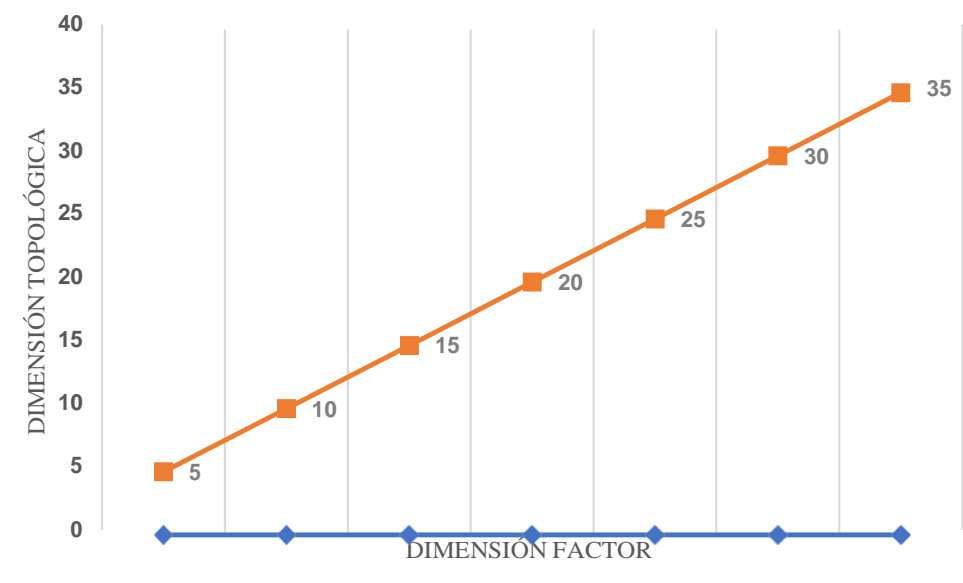

Nota: Esta figura representa la dimensión fractal en términos de estrategias para la gestión compleja. Elaboración propia.

Como se puede ver en la Figura 1, la dimensión fractal se entiende como el número de estrategias (autómata celular) necesarias para responder a los puntos diferenciales no idénticos. Es decir, en una organización constituida por cinco (5) áreas funcionales con un factor de dos (2) variables en una escala exponencial que incrementa una unidad, el sistema necesita para el primer período cinco (5) estrategias para responder a las necesidades del entorno. En ese sentido, el análisis muestra que en la medida en que incrementa las variables de un sistema, así mismo en cada período incrementa el número de estrategias a implementar. Por lo tanto, son estrategias que cambian de estado cuando interactúan con sus aledaños, en su diseño contienen una visión total de la(s) necesidad(es) de la organización y el estado de su ocurrencia se da como un sistema vivo, es decir, participa de la complejidad de los demás sistemas y realiza cambios acordes con los procesos de la organización y su relación con el entorno.

Por consiguiente, es una gestión que desde una conciencia ecológica ${ }^{11}$ se preocupa por superar los problemas que enfrenta la organización en su relación con el entorno (economía-naturaleza-medio ambiente) para honrar, respetar y colaborar con la naturaleza desde la posibilidad de comprender sus capacidades para mantener la vida y la sostenibilidad de las sociedades (Capra, 1996). De ahí nacen fenómenos de adaptación y supervivencia

11 E. Odum (1997) y R. Margalef (1974) presentan las diferencias entre el conservacionismo y preservacionismo en términos dela ecología. todos los casos, pero con el apoyo de las estrategias como
autómata celular se pretende responder a los grados de diferencia que hacen que el fenómeno sea distinto, sin que la naturaleza de la organización se pierda. 
que en la organización responden a comportamientos no periódicos y recurrencia de patrones a escalas espaciales y temporales.

En síntesis, es una organización disipativa que no vive de comportamientos estáticos, sino de múltiples rostros causantes de problemas sociales que confluye en la necesidad de una solución y que a partir de las estrategias como autómata celular se establece el límite de "supervivencia" y el "limite de muerte" del sistema. Por lo tanto, la gestión compleja permite conocer los segmentos pequeños y grandes y el límite de lo ilimitado de las actividades que ayudan a optimizar la(s) función(es) de la organización (Chávez Grimaldi, 2006). En ese sentido, la teoría fractal se convierte en una herramienta válida para el estudio de fenómenos dinámicos y no-lineales acorde a la complejidad del sistema, pero también a través de modelos computacionales de sistemas sociales (modelos basados en agentes) es posible explicar la complejidad del sistema y las necesidades sociales y ambientales que la rodean; en otras palabras, por medio del modelamiento ${ }^{12}$ y la simulación es posible lograr recrear las condiciones necesarias para la continuidad y adaptación del sistema.

\section{Conclusiones}

En la organización como estructura disipativa no tienen cabida la predicción ni el determinismo, así como tampoco existe una única medida de complejidad para el sistema. Se entiende que el sistema se caracteriza por la inestabilidad y el desequilibrio (no-equilibrio) en escenarios de complejidad creciente, la cual se busca absorber con las ciencias de la complejidad. ${ }^{13}$ De ahí nace la posibilidad de observar cosas distintas para adoptar escalas diferentes, según un modelo "multiescalar" de la realidad donde existe más de un observador. En este artículo se demuestra que la organización como estructura disipativa es un sistema "inteligible" y que aprende de las interacciones con los demás sistemas que, a su vez, se robustecen a través de redes, alianzas y cooperación para absorber la complejidad. Esto significa que el tiempo se convierte en un factor determinante en la complejización del sistema, que está condicionado en lo natural y se corresponde exactamente en la ausencia de equilibrio, o lo que es equivalente, en el carácter inestable o dinámico del equilibrio (Maldonado Catañeda, 2005).

Desde este punto de vista conceptual, se muestra la gestión compleja como el canalizador y catalizador de procesos inestables fruto de la interacción (comportamientos) entre los sistemas. Se evidencia también que a partir de las estrategias como autómata celular se logra testificar la solidez de la organización respecto a su adaptación y coevolución con el medio. Los datos que se recogen para la construcción de las estrategias se convierten en ayuda de aprendizaje para mejorar la inteligencia, las capacidades y la toma de decisión, que luego es evaluada frente a las condiciones, escenarios y situaciones de un entorno cada vez más complejo (Wang, Lingxi, Youg, Peijun, Fei-Yue, 2016).

\section{Referencias}

Arroyo Méndez, Millán y Hassan Collado, S. (2007). Simulación de procesos sociales basada en agentes software. Revista de metodología de ciencias sociales, 14, 139161.

Assmann, H. (2002). Placer y ternura en la educación. Hacia una sociedad aprendiente. Madrid: Narcea, S. A. De ediciones.

Bar-Yam, Y. (1997). Dynamics of complex systems. (P. Press, Ed.)

Bar-Yam, Y. (2002). General Features of Complex Systems. in Encyclopedia of Life Support Systems. EOLSS, UNESCO, EOLSS Publishers, Oxford.

Bateson, G. (1993). La unidad sagrada. Pasos hacia una ecología de la mente. Barcelona: Gedisa.

Bondarenko Pisemskaya, N. (Agosto de 2007). El lenguaje y la teoría del caos. Scielo, 23(53).

Briggs, J. y. (2005). Espejo y reflejo: del caso al orden. Guía ilustrada de la teoría del caos y la ciencia de la totalidad. (4 Ed ed.). Barcelona, España: Gedisa.

Cabrera Cuevas, J. (2009). Creatividad hoy. Una evolución hacia mayores niveles de conciencia y complejidad. Educación y futuro, 15-42.

Cabreras Cuevas, J. (2018). Epistemología de la creatividad desde un enfoque de complejidad. Educación y humanismo, pp. 113-126.

Capra, F. (1996). El punto crucial (Segunda ed.). Buenos aires: Editorial estaciones.

Chávez Grimaldi, O. M. (2006). La enfermedad: Una visión desde la teoría del caos y de los fractales. Medicrit, revista de medicina interna y medicina crítica, 3(3), 78-84.

Cornejo Álvarez, A. (2004). Complejidad y caos: guía para la administración del siglo XXI.

Eschenhagen y Maldonado, M. (2014). Un viaje por las alternativas al desarrollo. Universidad del Rosario.

Etkin, J. (2006). Gestión de la complejidad en las organizaciones: estrategias frente a lo imprevisto y lo impensado (1a Ed ed.). Buenos aires: Ediciones Granica S.A.

Gil Domínguez, J. (2006). La gestión empresarial bajo el enfoque de las organizaciones inteligentes en la sociedad de la información. NEGOTIUM2,6.

Hernandez Santana, A. (1997). El tejido interactivo de la organización laboral: la dualidad formal-informal. Centro de investigaciones Psicológicas y Sociológicas. Obtenido de http://bibliotecavirtual.clacso.org.ar/Cuba/ cips/20120827035902/cips4-1.pdf

Hock, D. (2001). El nacimiento de la era caórdica. Barcelona: 
Granica.

Kauffman, S. (2000). Investigations. Oxford: Oxford University Press.

Lozano y Fuentes, M. (01 de Junio de 2008). Tratamiento borroso del intangible en la valoración de empresas de internet. Obtenido de http://www.eumed.net/cursecon/ libreria/

Luhmann, N. (1998). Complejidad y modernidad: de la unidad a la diferencia. Valladolid: Trotta.

Luhmann, N. (2007). La sociedad de la sociedad. Ciudad de méxico: Universidad iberoamericana-Herder.

Maldonado Castañeda, C. (2003). El problema de la filosofía del conocimiento y el estudio de los sistemas complejos. Praxis filosófica, 17, 130-120.

Maldonado Catañeda, C. (2005). Complejidad de las ciencias y ciencias de la complejidad (1era ed.). Universidad Externado de Colombia.

Manrique Solana, R. (1987). Estructuras disipativas. De la termodinámica a la psicoterapia familiar. Asociación Especializada Neurosiquiatria, VII(22).

Max-Neef, M. (1993). Desarrollo a Escala Humana. Conceptos, aplicaciones y algunas reflexiones. Montevideo: Editorial Nordan-Comunidad.

Morin, E. (1993). El método. I. La naturaleza de la naturaleza. Madrid: Cátedra.

Morin, E. (2006). El método III. El conocimiento del conocimiento. Madrid: Ediciones cátedra (Grupo Anaya, S. A.).

Morris B, H. (2003). Dinamic open complex adaptive system. Tomado de tesis: Mercadeo con base en las ciencias de la complejidad y la lógica difusa. Universidad del Rosario.

Morris, H. (2003). Mercadeo con base en las ciencias de la complejidad y la lógica difusa. Tesis. Universidad del Rosario.

Negash, S. (2004). Business Intelligence. Communications of the Association for Information Systems, 13.
Nonaka y Takeuchi, I. (1999). La organización creadora de conocimiento. Cómo las compañías japonesas crean la dinámica de la innovación. Oxford University Press.

Porskein, B. (2004). Del ser al hacer: los orígenes de la biología del conocer. Santiago de chile: J.C. Sáenz.

Prigogine y Stengers, I. (1983). Naturaleza y creatividad. ¿Tan solo una ilusión? Una exploración del caos al orden. Tusquets.

Prigogine y Stengers, I. (2004). La nueva alianza. Metamorfosis de la ciencia. Madrid: Alianza Editorial, S.A.

Prigogine, I. (1997). El fin de las certidumbre, s. 1. Santillana.

Sáenz de cabezón, E. (11 de Noviembre de 2015). ¿Qué son los fractales? Obtenido de [Archivo de video]. Youtube. https://www.youtube.com/watch?v=Wea_1L-C9Xo

Senge, P. (1990). La quinta disciplina, s. I. Granica Española. Villalba Puerta, M. E. (Enero-junio de 2012). Gestión con base en las ciencias de la complejidad: Las organizaciones como estructuras disipativas. Universidad \& Empresa, 14(22). Obtenido de https://revistas.urosario.edu.co/ index.php/empresa/article/view/1960

Wagensberg, J. (1986). Proceso al azar. Barcelona: Tusquets. Wang, Lingxi, Youg, Peijun, Fei-Yue, X. (2016). ACP-based social computing and parallel intelligence: Societies 5.0 and beyond. ScienceDirect. CAAI Transactions on Intelligence Technology, 377-393.

Zimmerman, B. (Junio 19-22 de 1993). Chaos \& Nonequilibrium: the flip side of strategic porcesses. Organization Development Journal, s.d. 